\title{
Experimental Investigation of Defects Inspection for FRP Pipeline based on Single-side NMR
}

\author{
YANG Yong ${ }^{1}$ \\ ${ }^{1}$ Technology Inspection Center, Shengli Oil Field, \\ Dongying 257000, P.R. China \\ LIU Chao $^{1}$ \\ ${ }^{1}$ Technology Inspection Center, Shengli Oil Field, \\ Dongying 257000, P.R. China \\ WANG Guan-jun ${ }^{1}$ \\ ${ }^{1}$ Technology Inspection Center, Shengli Oil Field, \\ Dongying 257000, P.R. China
}

\author{
LIAO Guang-zhi ${ }^{2}$ \\ ${ }^{2}$ State Key Laboratory of Petroleum Resources \\ and Prospecting, China University of Petroleum, \\ Beijing102249, P.R. China \\ TAN Xiao-lin ${ }^{1}$ \\ ${ }^{1}$ Technology Inspection Center, Shengli Oil Field, \\ Dongying 257000, P.R. China \\ CHEN Kai \\ ${ }^{1}$ Technology Inspection Center, Shengli Oil Field, \\ Dongying 257000, P.R. China \\ ${ }^{2}$ State Key Laboratory of Petroleum Resources \\ and Prospecting, China University of Petroleum, \\ Beijing102249, P.R. China
}

\begin{abstract}
The fiberglass reinforced plastic (FRP) pipelines have been used widely in oil-gas gathering and transportation. The defects of FRP pipelines would increase with the extension of service time. However, it is very difficult to detect the defects of FRP pipelines on-spot quickly. In this paper, a new method detecting defects for FRP pipes has been provided based on the NMR. The proton density distributions have been obtained at different depth of FRP components using single-side NMR. The experimental results show that there is a significant change of proton density distribution at the location of defects. And, these results would be useful for defects inspection of composite material component.
\end{abstract}

Keywords: Mobile Single-Side NMR, FRP (fiber reinforced plastics) Pipes, Defects, Non-destructive Testing

\section{Introduction}

Fiberglass reinforced plastic (FRP) pipelines have been used widely in oil-gas gathering and transportation and water injection lines because of a series of advantages such as corrosion resistance, fatigue resistance and good economic benefit [1]. However, with the prolonging service time of FRP pipelines, the factors such as chemical corrosion and mechanical damage will result in the emergence of the pipeline defects, and accordingly the leakage accidents caused by the pipeline defects also occur frequently. To prevent the occurrence of the accidents, detection on the defects of FRP pipelines is very necessary. At present, the defects of the composite components can be detected using many technologies such as infrared thermal imaging and ultrasonic $\mathrm{C}$ scanning, but rapidly detecting the defects of the on-site FRP pipelines is still a difficulty to be solved in the field of detection technology [2].

The defects of FRP pipelines are mainly reflected from the perforation points caused by fiber delaminating layer or internal defects and the micro-cracks caused by pipe bending [3]. There are large amounts of hydrogen nuclei in FRP, and therefore, the hydrogen nuclei distribution in FRP will change once the FRP has 
defects. Also, the transmission mediums in FRP pipelines commonly include oil, gas, and water containing hydrogen, and the defects of the pipelines will be filled by the hydrogen-contained mediums and the amount of hydrogen nuclear in defects will obviously change. Therefore, measuring the change of the hydrogen amount in FRP pipelines and detecting their defects are feasible using magnetic resonance (NMR).

NMR, as a rapid non-destructive testing technology, has been widespread applied [4, 5]. Sardashti and Blumich used to make an experiment for detecting the defects (e.g. composite deformation and fatigue) of $\mathrm{PE}$ pipelines using the low-field NMR technology [6, 7]. References $[6,7]$ show that NMR can be applied to detect the deformation and fatigue of composite materials. Based on the above reasons, NMR is introduced to detecting the defects of FRP pipelines and also a related study is conducted, and this is the purpose of this paper.

\section{Materials and methods}

\subsection{Experimental instrument}

In Fig.1, low-field NMR unilateral magnetic resonance device (NMR-Mouse) produced by German Magritek Company was used for the experiment, and its resonance frequency was $13 \mathrm{MHz}$. Sensor was placed in a bracket installed with a controllable stepper motor and could accurately position the distance of the sensitive area of the measured samples from the surface of the magnet, so as to determine the depth of the samples. Because single-side NMR device is designed open, its detecting work is not limited by the size and shape of the measured samples.

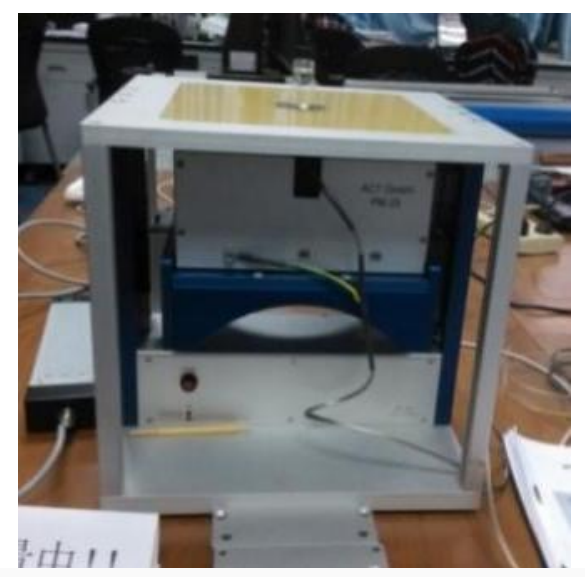

Fig.1 the single-side NMR device for Experiment

\subsection{Experimental samples}

Due to the limitation of processing technology, micro defects of the pipelines were difficult to process in the laboratory. Thus, three sections of FRP pipelines containing micro cracks were used as samples for the experimental detections. The length and diameter of these samples were 350 $\mathrm{mm}$ and $89 \mathrm{~mm}$ respectively. As shown in Fig.2, sample 1 contained a perforation point that was visible; sample 2 contained a micro perforation point that was calibrated by a short-term hydraulic experiment; sample 3 contained a micro crack that was determined by a hydraulic experiment.

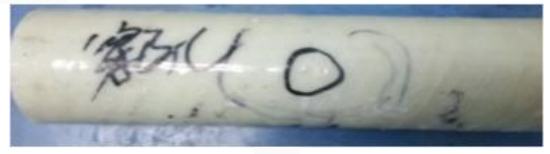

(a)

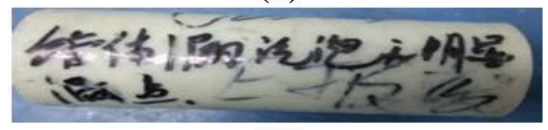

(b)

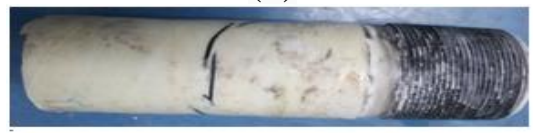

(c)

(a) Perforation point; (b) micro leak point; (c) micro crack

Fig.2 the samples with micro defects 


\subsection{The measurement of free induction decay signal}

Samples to be detected were placed within the magnetic field of NMR-MOUS device magnet excitation, and control instrument was used for scanning and detecting free induction decay (FID) signal and spin-echo sequence (SE) pulse. In the experiment, FID and CPMG signal acquisition parameters were set as follows: $\mathrm{D}_{1}=0.06 \mathrm{~ms}$ (interval between $90^{\circ}$ and $180^{\circ}$ pulses), $\mathrm{D}_{0}=500 \mathrm{~ms}$ (pulse repetitive sequence time), sampling resonance frequency $=13 \quad \mathrm{MHZ}$; sampling points $=35$; echo quantity $\mathrm{C}_{0}=128$; repeated scanning times $\mathrm{Ns}=256$.

To identify and locate the defects, relative nuclear hydrogen proton densities of different positions $(5 \mathrm{~cm}, 15 \mathrm{~cm}, 20 \mathrm{~cm}$ and $25 \mathrm{~cm}$ ) away from one end of samples were measured respectively. In addition, the density distribution of protons at different depth away from the surface of samples was measured, and the slice thickness of each layer was $0.4 \mathrm{~mm}$ and the quantity of slices was 12, as shown in Fig.3.
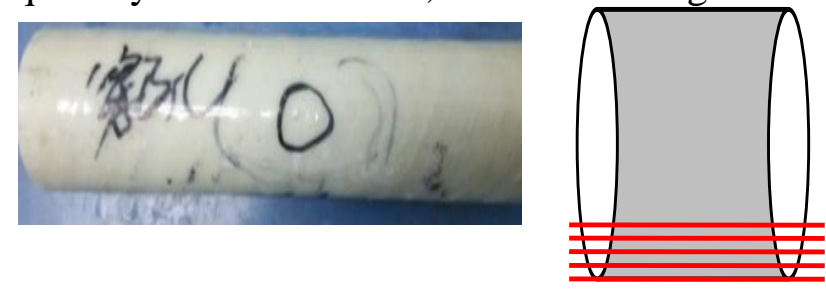

Fig.3 The schematic diagram of sample slice

\subsection{Experiment principle}

To rapidly detect the defects of FRP pipelines, the application of FID signal to the analysis is beneficial. In general, FID is an exponential decay signal, and transverse relaxation time $\mathrm{T}_{2}$ can be solved using the signal. For FRP material, its FID signal can be represented as follows: $v \propto M_{z} \sin \theta \cos \left(\omega_{0} t\right) \exp \left(-t / T_{2}\right)$

Where,

$M_{z}$ _ the magnetization vector with the same direction to externally-added static magnetic field; $\theta$ - the angle of ${ }_{M}$, to deviate from static magnetic field $\mathrm{B}$;

$\omega_{0}$ _the Larmor frequency;

$T_{2}$ _transverse relaxation time;

In this experiment, the FID signal tested by NMR was used for the fitting, and $T_{2}$ was solved according to the fitting formula, as shown in Fig.4 (black curve - FID signal; red curve - the fitting curve). 1/T2 of different detection points was drawn on a curve, and thus, the relative hydrogen nuclei density distribution at different location was obtained.

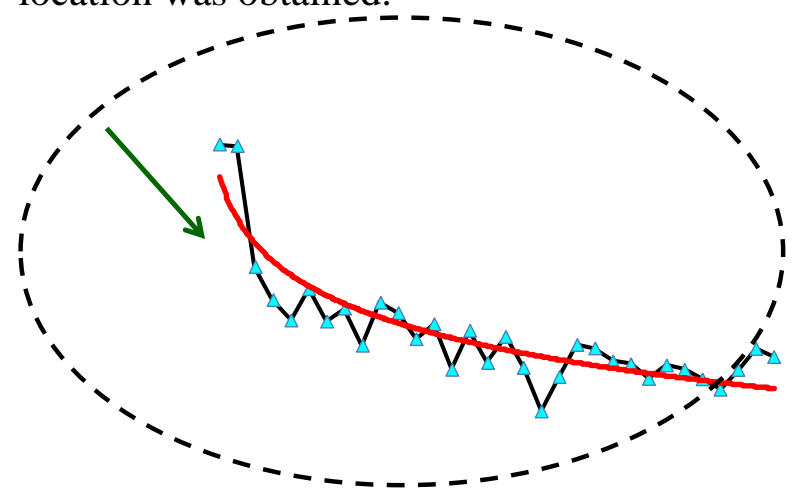

Fig. 4 FID signal and its fitting curve

\section{Results and analysis}

The proton density distribution of sample 1 containing a perforation point was measured, and the result was shown in Fig.5: horizontal ordinate -the length of the sample; vertical coordinate$1 / T_{2}$, indicating the relative hydrogen proton density distribution of the sample. The measured data insignificantly changed at locations $5 \mathrm{~cm}$, $15 \mathrm{~cm}$ and $30 \mathrm{~cm}$, and therefore, the hydrogen atom amount did not significantly change at these locations, so these locations were without defects. From the measured results at locations $20 \mathrm{~cm}$ and $25 \mathrm{~cm}$, it was known that $1 / \mathrm{T}_{2}$ amplitude significantly changed with the change of the measured depth, and this result was consistent with that of locating defects using short-term hydrostatic test. The significant change of defect $1 / T_{2}$ was because the average size of the non-crystalline state domain was reduced, 
namely, the hydrogen proton density distribution decreased at the defect.

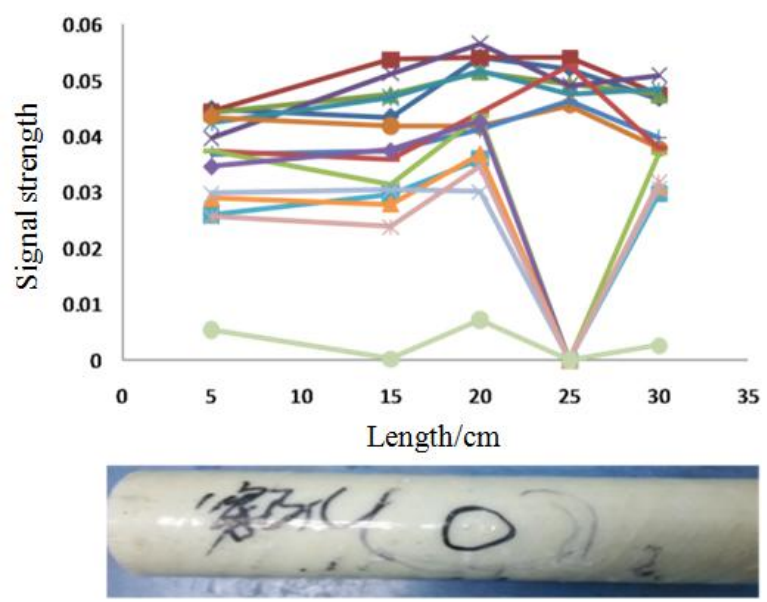

Fig.5 the test results of NMR for the sample with perforation point

The result of measuring sample 2 with micro leak point was shown in Fig.6. Similar to the result of Fig.5, NMR test result showed that the change of the proton density distribution of the measured sample was significant at the location $15 \mathrm{~cm}$ away from the left end of the sample as the detected depth was different. Consistent with the short-term hydraulic pressure result, the location with a significant change of proton density was where micro leak point existed. From the proton density distribution measured at different depth at the location $15 \mathrm{~cm}$ in Fig.6, the defect perforated the pipeline wall. Similarly, the reduction of $1 / \mathrm{T}_{2}$ amplitude was because the amount of the hydrogen atoms at the location of the sample's micro defect 1 changed. The results of the above two experiments indicate that the perforation defects of FRP pipeline can be rapidly detected and positioned using low-field NMR technology.

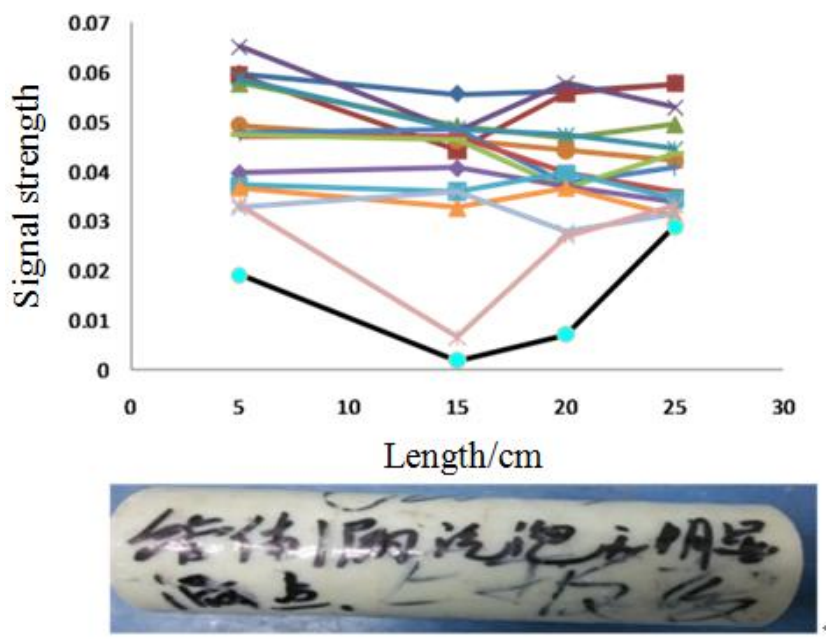

Fig.6 The test results of NMR for the sample with micro leak point

In Fig.7, the result of measuring sample 3 containing micro crack using NMR-MOUSE was shown. Analyzed from the NMR results of five locations at different depth, the change of the proton density distribution at the corresponding micro crack location was extremely significant along with the change of the measured depth. The proton density of each layer changed at depth $20 \mathrm{~cm}$, namely, micro crack perforated the entire pipeline. The defects could be can be accurately positioned at $\mathrm{T}_{2}$ using single-side NMR technology, but micro crack NMR detection result was insignificantly different from perforation point detection result, namely, the proton density distribution measured using NMR was difficult to distinguish the types of defect. A deeper data analysis or NMR imaging is required if the types of the defects of FRP pipelines are necessary to distinguish. 


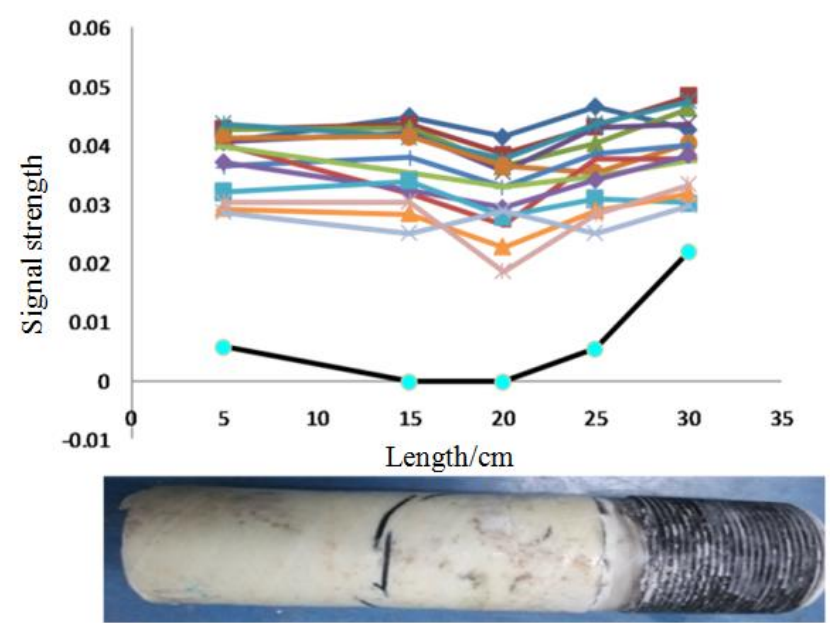

Fig.7 the test results of NMR for the sample with micro crack

\section{Conclusion}

In this paper, the defects of FRP pipelines were experimentally detected using single-side NMR, and the results are concluded as follows:

First, the defects of FRP pipelines can be rapidly detected using single-side NMR.

Second, the proton density distribution significantly changes at the location of defects. Therefore, a defect can be accurately located by measuring the proton density distribution, and also the depth of defect can be defined through multi-layer scanning.

Third, a simple FID attenuation curve is still difficult to distinguish between the types of defect, even though NMR results can provide the information about the location and depth of defect.

\section{References}

[1] LI Ze-quan, SU Wei-gang, LI Wei. Construction Technique and Quality Control of Fiberglass Reinforced Plastic Pipeline [J]. Petroleum Engineering Construction, 2006, 32 (3): 48-50.

[2] Liu Wenxia, Shen jingling, Liu Yingtao, et al. Nondestructive Testing for FRP Skin Paper Honeycomb Material [J]. Non-destructive Testing, 2009， 31 (12): 990-992.

[3] Dong Zhiheng, Luo Jun, Li Xueqiang,et al. The Summary of Failure Analysis and Test Procedure for FRP Pipe [J]. Guangzhou Chemical Industry, 2013, 40 (14):107-108.

[4] Zhou Hua, Li Yingliang, Gao Feng, et al. Evaluation of Consolidation of The Brick Materials by Mobile Single-side NMR [J]. Journal of Building Materials, 2013, 16 (6):1097-1102.

[5] Xiong Ting, Zhang Yingli, Cai Qing. Detection of Sugar Solution based on Low-field Magnetic Resonance Technology [J]. Journal of China University of Metrology, 2013, 24 (3): 219-223.

[6] B. Blumich, F. Casanova, A. Buda, et al. Mobile NMR for Analysis of Polyethylene Pipes [J]. ActaPhysicaPolonica A, 2005, 108 (1):13-23.

[7] M. Sardashti, B.A. Baldwin and D.J.O'Donnell. NMR Imaging of Polyethylene Pipes [J]. Journal of Polymer Science: Part B. Polymer Physics, 1995, 33: 571-576. 